\title{
The Prevalence of Coxofemoral Osteoarthritis in Small Dog Breeds and HD Screening in Cairn Terries A Clinical Radiological Study Jens Arnbjerg*
}

Department for Diagnostic Imaging, Faculty of Health and Medical Sciences, University of Copenhagen, Dyrlaegevej 32, DK 1870 Frederiksberg C, Denmark

Received: 15 April, 2017; Accepted: 12 July, 2017; Published: 24 July, 2017

*Corresponding author: Jens Arnbjerg, Department for Diagnostic Imaging, Faculty of Health and Medical Sciences, University of Copenhagen, Dyrlaegevej 32, DK 1870 Frederiksberg C, Denmark, Tel:+45 43967126; Fax:+45 35282929; E-mail: jarn@image.dk

\begin{abstract}
Background: The prevalence of coxofemoral osteoarthritis being very high, $>40 \%$, in some large dog breeds. The aim of the study was to estimate the prevalence of osteoarthritic changes in the hip joints in small dog breeds and study the clinical relevance of Hip Dysplasia scoring in Cairn Terries in Denmark.
\end{abstract}

Methods: In a five-year-period study of 2423 small dog breeds $(<15 \mathrm{~kg})$ at the University Hospital, Copenhagen the prevalence of osteoarthritis in the hip joints was calculated

A full clinical examination was performed and hip joint radiographs taken in 18 Cairn Terriers, earlier officially scored for hip dysplasia by The Danish Kennel Club, 13 of them with a score of C, D or E respectively.

Results: The prevalence of coxofemoral osteoarthritis in small dog breeds was found to be $0.5 \%$, which is in accordance with earlier published studies in small dog breeds in other countries.

In 18 Cairn Terriers, no correlation was found between the initial Hip Dysplasia scoring and clinical signs indicating discomfort. No evidence of osteoarthritis and no progression of hip joint laxity or deformation could be found on the follow up radiographs taken $>18$ months (mean. 38 months) after the initial radiological examination evaluated for official HD screening.

Conclusion: Due to the very low prevalence in osteoarthritis in small dog breeds and low clinical significance of the radiological scoring of the hips in Cairn Terrier according to parameters set by FCI it cannot be recommended to use the same parameters in Cairn Terrier as in large dog breeds in routine screening programmes for Hip Dysplasia in Cairn Terriers.

Keywords: Hip Osteoarthritis; Cairn Terrier; HD in small dog breeds; Prevalence coxofemoral osteoarthritis; Hip Dysplasia scoring in Cairn Terrier

\section{Introduction}

Hip Dysplasia has for the last 50 years been a major consideration in the discussion of hind leg lameness among the larger dog breeds. Hip Dysplasia screening programmes have intensified this discussion and have extended to involve the smaller dog breeds even though the smaller dog breeds have few clinical problems with hind leg lameness with exception of very few breeds as earlier shown in several studies.

Different authors have estimated the prevalence of Hip Dysplasia in different breeds. Estimates range from $59.7 \%$ in Cane Corso, $43.2 \%$ in Saint Bernhard to $4.2 \%$ in the Siberian Husky and in the smaller breeds Miniature Schnauzer: $1.5 \%$ and $3.2 \%$ is reported in the Dachshund [1-3]. The discussion of the clinical relevance of Hip Dysplasia for the individual dog has always been going on, as there is not always good correlation between the radiological changes found and the clinical importance for the individual dog. Even if a dog has significant Hip Dysplasia sign e.g. sublimation on the radiographs it is not always so, that osteoarthritis will develop later. The different scoring systems used in the different continents are other confusing elements [4]. Recently a study showed very significant laxity in hip joints in some small dog breeds without secondary developed osteoarthritis [5].

The aims of the study were

1) To estimate the prevalence of osteoarthritic changes in the hip joints of small pure breed dogs in Denmark and,

2) To correlate the hip score on radiographs with the clinical condition in Cairn Terries in Denmark, earlier scored according to the Fédération Cynologique Internationale (FCI) criteria used routinely by The Danish Kennel Club. The Cairn Terrier race was chosen as a dog-race with very limited problem with orthopaedic problems and a breed with relative high number being screened by DKK. 


\section{Material \& Methods}

\section{The prevalence study}

For presence of osteoarthritis changes (malformation of femoral head, acetabulum, sclerosis, periarticular new bone formation) was performed retrospectively on radiographs of pure- bred small dogs ( $>1$ year of age, body weight $<15 \mathrm{~kg}$ ) taken at the Small Animal Hospital at University of Copenhagen in a period of 5 years (2006 - 2010). During this period, a total of 2423 small pure breed dogs were presented for all kind of consultation. From these 229 Ventro-Dorsal radiographs of the pelvic area from 37 different pure breeds were included in the study (Table 1).

\begin{tabular}{|c|c|c|c|c|c|c|c|}
\hline \multirow{2}{*}{ Breed } & \multicolumn{2}{|c|}{ Osteoarthritis } & \multirow{2}{*}{ Total } & \multirow{2}{*}{$\begin{array}{c}\text { Osteoarthritis } \\
\text { among dog } \\
\text { X-rayed }\end{array}$} & \multirow{2}{*}{$\begin{array}{c}\text { Total no at } \\
\text { risk }\end{array}$} & \multirow{2}{*}{$\begin{array}{l}\text { Osteoarthritis } \\
\text { Among of dog at } \\
\text { risk }\end{array}$} & \multirow{2}{*}{$\begin{array}{c}\text { Confidence } \\
\text { interval } \\
(a=0.05)\end{array}$} \\
\hline & Yes & No & & & & & \\
\hline Beagle & 0 & 6 & 6 & $0.0 \%$ & 123 & $0.0 \%$ & - \\
\hline Cairn Terrier & 1 & 26 & 27 & $3.7 \%$ & 187 & $0.5 \%$ & $(-0.0051 ; 0.0158)$ \\
\hline $\begin{array}{l}\text { Cavalier king charels } \\
\text { spaniels }\end{array}$ & 1 & 9 & 10 & $10.0 \%$ & 226 & $0.4 \%$ & $(-0.0042 ; 0.0131)$ \\
\hline Chihuahua & 1 & 15 & 16 & $6.3 \%$ & 212 & $0.5 \%$ & $(-0.0045 ; 0.0716)$ \\
\hline Coton de Tulear & 1 & 4 & 5 & $20 \%$ & 41 & $2.4 \%$ & $(-0.0228 ; 0.0716)$ \\
\hline Dachshound & 0 & 34 & 34 & $0.0 \%$ & 419 & $0.0 \%$ & - \\
\hline $\begin{array}{l}\text { Danish-Swedish } \\
\text { Farmdog }\end{array}$ & 0 & 17 & 17 & $0.0 \%$ & 279 & $0.0 \%$ & - \\
\hline French Bull dog & 3 & 9 & 12 & $25 \%$ & 158 & $1.9 \%$ & $(-0.0023 ; 0.0403)$ \\
\hline Maltese & 0 & 5 & 5 & $0.0 \%$ & 21 & $0.0 \%$ & - \\
\hline Miniature Schnauzer & 0 & 5 & 5 & $0.0 \%$ & 68 & $0.0 \%$ & - \\
\hline Papillor/phalene & 0 & 9 & 9 & $0.0 \%$ & 76 & $0.0 \%$ & - \\
\hline $\begin{array}{l}\text { Parson and Jack } \\
\text { Russel Terriers }\end{array}$ & 0 & 11 & 11 & $0.0 \%$ & 147 & $0.0 \%$ & - \\
\hline Pug & 1 & 5 & 6 & $16.7 \%$ & 88 & $1.1 \%$ & $(-0.0108 ; 0.0925)$ \\
\hline Shetland Sheepdog & 2 & 5 & 7 & $28,9 \%$ & 51 & $3.9 \%$ & $(-0.0141 ; 0.0116)$ \\
\hline $\begin{array}{c}\text { West Highland White } \\
\text { Terrier }\end{array}$ & 1 & 15 & 16 & $6.3 \%$ & 254 & $0.4 \%$ & $(-0.0038 ; 0.0116)$ \\
\hline Yorkshire Terrier & 0 & 6 & 6 & $0.0 \%$ & 73 & $0.0 \%$ & - \\
\hline Total & 11 & 181 & 192 & $4.7 \%$ & 2423 & $0.5 \%$ & $(-0.0019 ; 0.0072)$ \\
\hline $\begin{array}{l}\text { Other Small breeds } \\
\text { (21 breeds) }\end{array}$ & 2 & 35 & 37 & $5.4 \%$ & $\mathrm{NC}$ & $\mathrm{NC}$ & $\mathrm{NC}$ \\
\hline Grand total & 13 & 216 & 229 & $5.7 \%$ & $\mathrm{NC}$ & $\mathrm{NC}$ & $\mathrm{NC}$ \\
\hline
\end{tabular}

Table 1 shows the material of dogs on which the prevalence's are calculated and organized in relations to different breeds and the prevalences in the different breeds showing the average prevalence to be $0.5 \%$. The Shetland Sheepdogs and the French bulldog hawing the highest individual prevalence, 3.9 and 1.9 respectively. Dachshound, Miniature Schnauzer, Papillon and Yorkshire Terrier having $0.0 \%$ in this material.

To be able to evaluate if there is an age or gender related prevalence of osteoarthritic changes, the dogs were separated in 4 groups (12 - 35 months and > 36 months and gender groups).

The age and clinical findings were recorded. Dogs were excluded if affected by Legg-Calve- Perthes disease, femoral head excision or a fracture or luxation of the pelvic bones. If the same dog was examined more than one time, the latest result was used. Poodle (different sizes) and other breeds were excluded to prevent an imbalance in the material, if The Danish Kennel Club re- 
quires a screening program for Hip Dysplasia before using that specific breed for breeding.

Calculation of the p-value was done in $\mathrm{R}$ version 2.10.1 (2009-12-14), available at http://www.r-project.org/ and the null hypothesis was rejected when $\mathrm{p}$ was $<0.05$ (Table 1 ).

\section{Follow up clinical signs of Hip Dysplasia}

A questionnaire was sent to all Cairn terrier owners who had their dogs evaluated by The Danish Kennel Club for hip dysplasia from 2008 -2012. The questions were related to clinical performance, specifically the dog's gait and if it had had any chronic pain related to the hip. The first radiological evaluation made by The Danish Kennel Club's special panel (DKK) as a service outside screening programs prerequisite for breeding in other breeds. In that study 13 (72\%) of the 18 dogs had scores in the categories C, D \& E.

The questionnaire was returned from 23 owners of 30 Cairn terrier dogs. All dogs were offered a new but the same radiographic examination of the hips as used for the DKK-screening, at the University Small Animal Hospital and 18 Cairn Terrier-dogs accepted the offer.

The Cairn terrier breed is not restricted from breeding without screening and none of the dogs were original radiographed and evaluated by the DKK due to clinical problems. The breeders only wanted to know what their dog hips looked like.

The dogs presented for new radiographs of the hips were examined clinically before the radiographs were taken. The dogs motion was recorded, especially any signs of lameness at a walk and at a trot; muscle tone and -mass were evaluated as well as the range of passive motion and signs of pain in the hip and sacroiliac joints. Standard V/D radiographs as used for the previously screening program were taken in dorsal recumbence under sedation. The images were compared with the original images read blind by 2 trained diagnostic imaging examiners and consensus was compiled. The dogs were between 2.8 years and 7.5 years of age, mean: 4.9 years, at the second examination. The interval between first and second evaluation was 18 to 49 months with a mean of 38 months. The first evaluation made by the DKK had scored $72 \%$ of the 18 dogs in the categories C, D \& E.

The new radiographs were examined with attention to any signs of coxofemoral osteoarthritic changes (malformation of Femoral head, Acetabulum and osteoarthritic changes e.g. sclerosis, periarticular new bone formation).

Due to DKK's security policy, the radiographs cannot be published; therefore no pictures are shown here.

Cairn terrier was chosen as a breed with limited orthopaedic problem and a reasonable number were examined for screening by the Danish Kennel Club, and the Cairn Terrier Club was interested in the project.

\section{Results}

\section{The prevalence study}

Out of the 229 small pure breed dogs used in this study,

13 (5.7 \%) showed secondary arthrotic changes. Considering the total number of pure breed small dogs (2423), the prevalence of secondary arthrotic changes on ventro/dorsal radiographs was $0.5 \%$ (95\% confidence interval). The prevalence was $4.3 \%$ for dogs aged 12 - 35 months and $6.3 \%$ for dogs aged $>36$ months. There was no statistically significant difference in prevalence of osteoarthritis between the two age groups as well as there was no significant difference between genders (Table 1).

Ten out of the 13 dogs with osteoarthritic changes were radiographed because of clinical pain in the hip joints, three showed no sign of pain. They were between 1 and 3.5 years old. 5 of them had subluxation but no altered conformation or pathological changes of the Femoral head and/or Acetabulum.

\section{Follow up clinical signs of Hip Dysplasia}

The aim of this part of the study was to correlate the hip score to the clinical condition evaluated more than 18 months after the first radiological pictures were taken.

Completed questionnaires concerned clinical condition was answered for 18 individual dogs earlier evaluated by the DKK (scored: $3: \mathrm{A} ; 2$ : B; 7: C; 4: D and 2: E) were returned. None of the dogs scored A, B and $E$ by the DKK had ever been lame and 4 from the group $C$ and $D$ had an episode of short period of lameness but none were diagnosed with hip related pain according to the questionnaire response.

All eighteen dogs were examined clinically at the day of re-radiographing at the University Hospital. The owners had no complains about actual pain. However, one dog showed pain in the pelvic area when the hind legs were abducted and extended, but no sign of disuse muscle atrophy was seen. The radiographs of this dog only showed subluxation of the hips.

The radiographs were all compared with those radiographs previously evaluated by The Danish Kennel Club. There were no differences observed between the original and the new radiographs irrespective of the interval between the first and second radiographic examination.

One dog showed signs of osteoarthritis on the radiographs ( 6 years of age) but no actual lameness or pain was found at the clinical examination at the day of the re-radiographic examination at the University Hospital.

As there was only one dog showing pain at the re-examination. There was no correlation between clinical examination and the official screening radiographic scores in these Cairn Terrier dogs.

\section{Discussion}

This prevalence study shows that Hip Dysplasia and osteoarthritis do occur in Denmark in a few small breed dogs. As earlier studies have shown only few of them develops clinical signs. The prevalence of coxofemoral osteoarthritis at $0.5 \%$ in small breed dogs found in this study correspond well with results found by Rettenmaier, et al. (Dachshund: $3.2 \%$ and Miniature Schnauzer: $1.5 \%$ and Witsberger, et al. West Highland White Terrier: $0.64 \%)[3,6]$.

This contrasts with overall prevalence among larger purebred dogs (19.7\%) and mixed breed dogs (17.7\%) according to Rettenmaier, et al. and the osteoarthritic-prevalence increases with increasing age in prevalence studies in large breed 
dogs but not in this material of small breed dogs $[3,7,8]$.

Swenson, et al found that the benefit of routine screening for Hip Dysplasia in small dogs is debatable [9].

The present clinical/radiological study supports this view since there was no clinical correlation in the Danish Cairn Terriers between the scoring applied by FCI/DKK and lameness found by the owners and at the clinical examination at the day of the second radiographic examination. Also, this study shows no significant progression of the severity of osteoarthritis neither clinically nor radiographically, even among dogs in the severe Hip Dysplasia scoring groups (D \& E) during the time span (minimum $1 \frac{1}{2}$ year) after the first evaluation. The minimum interval of minimum 18 months (mean was 38) is not a short period for evaluation of osteoarthritic lesions, as in larger breeds 1 year can make a big difference with increasing osteoarthrtitic changes and clinical symptoms of pain [8]. In the larger breed dogs, many studies have shown further development of osteoarthritis over the time span of one year, and most elderly dogs in larger breeds scoring D and $E$ show increasing clinical signs of pain if not treated permanently with painkilling drugs [10]. No publication is found to explain the difference in progression of degenerative joint disease in small and large dog breeds, but it is generally accepted that higher weight put more stress on joints than lower weight. The mean age of the Cairn Terrier was 4.9 years at the second evaluation. The mean interval of 38 months between evaluations should be enough time to allow development of osteoarthritis in dogs initially scored C, D or E. This is supported by Kealy, et al., Smith, et al., and Farrell, et al. who all found progression of osteoarthritis with time and Malm, et al. found significant increase of pain in dogs suffering from osteoarthritis after 12 months interval examinations most published studies of progression of osteoarthritis in the dogs are based on larger breeds and no publication concerning small breed dogs [7-11]. However, there are a few publications on the outcome of specific orthopaedic treatment gives better results in small breed dogs first of all due to weight control has an indirectly influence on the degenerative joint disease by reducing stresses on the joint [12-14].

A major reason for The Danish Kennel Club scoring these 18 Cairn terrier dogs so high could be the subluxation, as there are very limited changes in and around the joints and the other scoring parameters.

It has earlier been found that some subluxation and lower Norberg Angle in some breeds does not always result in degenerative joint disease $[5,12]$. The findings in this study with mild subluxation of the hips, but no subsequent periarticular changes, are in accordance with Culp, et al [12] who found the Norberg Angle as an inaccurate predictor of degenerative hip joint disease in 6 out of 7 breeds.

In the Cairn terrier and some other small breed dogs e.g. Dachhund and Pekinese, the conformation, position and relation between the Greater Trochanter, the Neck and the Head of Femur and the position of the Femoral Head in relation to acetabulum are different from what is seen in the larger breeds e.g. German shepherd and Retriever. In some small breed dogs, the Collum Femoris angle is more open than in the larger breeds. Therefore the use of other parameters should be created for screening, if screening should be made in Cairn Terrier having that small prevalence in osteoarthritis in the hip joints which is shown in all prevalence studies in small dog breeds.

However, there are also in this material a few breeds like Shetland sheepdog, Coton de Tulear and French bulldog which now and then develops osteoarthritis and they have a prevalence of $3.9,2.4$, and 1.9 respectively in this material. Even these breeds have a much less problems compared with most of the bigger dog breeds.

According to the prevalence in previous studies as well as in this study of Cairn Terrier, the clinical problem of HD in Cairn Terrier is not important. The established method of screening dogs for HD in the Cairn Terrier breed results in taking dogs out of breeding on false grounds as the Cairn Terriers very seldom develops clinical problems in their hips. It is shown clearly in this follow up study that none of the dogs showed clinical problems at any time when examined. The development of osteoarthritis could not be demonstrated on the new radiographs even the earlier official screening showed high HD-scores (C \& D).

\section{Conclusion}

\section{The prevalence of osteoarthritis in small dog breeds}

$(<15 \mathrm{~kg}$ ) in Denmark (0.5\%) was found to be like what is found by other authors in other countries.

\section{The follow up study in Cairn terrier}

Showed no correlation between the official score for Hip Dysplasia and the clinical findings as well as no aggravate clinical or radiological status were found after more than 18 months after the official HD scorings.

Therefore the official scoring by DKK of the hips do not seem to have a significant clinical importance in Cairn terrier neither for development of osteoarthritis or for development of clinical abnormal gait or pain.

Therefore other parameters should be taken into consideration, if Hip Dysplasia screening programs should be performed in most small dog breeds and especially in Cairn terrier as it is proved in this study.

\section{Acknowledgement}

The author wish to thank DVM, Stine Fuglede for her help with collecting the material and the statistical analyses.

\section{References}

1. Genevois JP, Remy D, Viguier E, Carozzo C, Collard F, Cachon T, et al. Prevalence of hip dysplasia according to official radiographic screening, among 31 breeds of dogs in France. Vet comp Orthop Traum. 2008;21(1):21-24. doi: 10.3415/VCOT-07-02-0014

2. Riser WH. The dog as a model for the study of hip dysplasia. Growth, form and development of the normal and dysplastic hip joint. Vet Pathol. 1975;12(4):229-334.

3. Rettenmaier JL, Keller GG, Lattimer JC, Corley EA, Ellersieck MR. Prevalence of canine hip dysplasia in a veterinary teaching hospital population. Vet Radiol Ultrasound. 2002;43(4):313-318. 
4. Flückiger M. Scoring radiographs for canine hip dysplasia - the big three organizations in the world. Eur J Comp Anim Pract. 2007;17(2):135140.

5. Arnbjerg J. Hip Joint Laxity in Small Dog Breeds. A Radiological Study. SOJ Vet Sci. 2017;3(1):1-5

6. Witsberger TH, Cook JL, Hahn AW, Schultz LG, Villamil JA. Prevalence of and risk factors for hip dysplasia and cranial cruciate ligament deficiency in dogs. J Am Vet Med Assoc. 2008;232(12):1818-1824. doi: 10.2460/javma.232.12.1818

7. Kealy RD, Ballam JM, Biery DN, Lawler DF, Lust G, Mantz SL, et al. Evaluation of the effect of limited food consumption on radiographic evidence of osteoarthritis in dogs. J Am Vet Med Assoc. 2000;217(11):1678-1680.

8. Smith GK, Biery DN, Lawler DF, Kealy RD, McKelvie PJ, Paster ER, et al Lifelong diet restriction and radiographic evidence of osteoarthritis of the hip joint in dogs. J Am Vet Med Assoc. 2006;229(5):690-693.

9. Swenson L, Audell L, Hedhammar A. Prevalence and inheritance of and selection for hip dysplasia in seven breeds of dogs in Sweden and benefit: cost analysis of a screening and control program. J Am Vet Med Assoc. 1997;210(2):207-214.
10. Farrell M, Arnott JL, Bennett D, Carmichael S, Clarke SP, Clements $\mathrm{DN}$, et al. Retrospective evaluation of the long-term outcome of nonsurgical management of 74 dogs with clinical hip dysplasia. Vet Rec. 2007;160(15):506-511.

11. Malm S, Bonnett BN, Egenwall A, Fikse F, Gunnarsson L, Hedhammar A, et al. Association between radiographic assessment of hip status and subsequent incidence of veterinary care and mortality related to hip dysplasia in insured Swedish dogs. Prev Vet Med. 2010; 93(23):222-232. doi: 10.1016/j.prevetmed.2009.09.017

12. Culp WTN, Gregor TP, Kapatkin AS. McKelvie PL, Powers MY, Smith GK. Evaluation of the Norberg angle threshold: a comparison of Norberg angle and distraction index as measures of coxofemoral degenerative joint disease susceptibility in seven breeds of dogs. Vet Surg. 2006;35(5):453-459.

13. Richardson DC, Schoenherr WD, Zicker SC. Nutritional management of osteoarthritis. Vet Clin North Am Small anim Pract. 1997;27(4):883911.

14. Berger B, Knebel J, Steigmeier-Reith S,Reese S, Meyer-Lindenberg A. Long-term outcome after surgical treatment of cranial cruciate ligament rupture in small breed dogs. Comparison of tibial plateau level- 